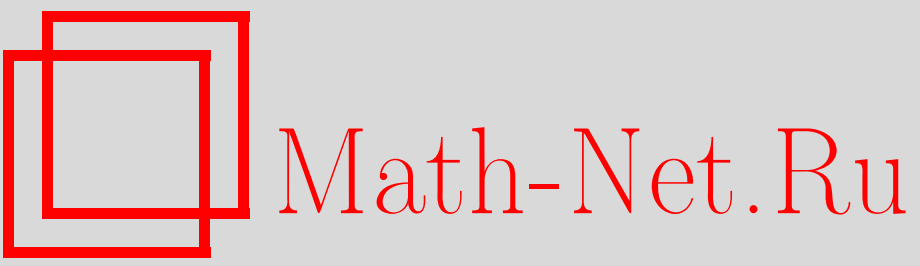

В. В. Сенатов, О квазинеравномерных оценках точности аппроксимации в центральной предельной теореме, Теория вероятн. и ее примен., 2010, том 55, Выпуск $3,432-445$

DOI: https://doi.org/10.4213/tvp4235

Использование Общероссийского математического портала Math-Net.Ru подразумевает, что вы прочитали и согласны с пользовательским соглашением

http://www.mathnet.ru/rus/agreement

Параметры загрузки:

IP : 52.6 .47 .48

26 апреля 2023 г., 10:55:05 


\title{
О КВАЗИНЕРАВНОМЕРНЫХ ОЦЕНКАХ ТОЧНОСТИ АППРОКСИМАЦИИ В ЦЕНТРАЛЬНОЙ ПРЕДЕЛЬНОЙ TEOPEME ${ }^{1)}$
}

\begin{abstract}
Предложены оценки скорости сходимости в центральной предельной теореме, которые можно назвать квазинеравномерными. Они имеют преимущества как перед равномерными, так и перед неравномерными оценками.
\end{abstract}

Ключевые слова и фразы: центральная предельная теорема, оценки скорости сходимости.

Цель этой работы - продемонстрировать оценки точности аппроксимации в центральной предельной теореме (ЦПТ), которые мы будем называть квазинеравномерными.

Пусть $X_{1}, X_{2}, \ldots$ - независимые случайные величины, имеющие общее распределение $P$ с нулевым средним и единичной дисперсией, для которых третий абсолютный момент $\beta_{3}=\mathbf{E}\left|X_{1}\right|^{3}$ конечен. Обозначим $P_{n}$ распределение нормированной суммы $\left(X_{1}+\cdots+X_{n}\right) n^{-1 / 2}, \Phi-$ стандартный нормальный закон и $\varphi(x)=e^{-x^{2} / 2} / \sqrt{2 \pi}-$ его плотность. Пусть для данного числа $n$ существует плотность $p_{n}$ распределеления $P_{n}$. Нас интересует вопрос об оценке величины $\left|p_{n}(x)-\varphi(x)\right|$. Для нее известны как равномерные оценки, которые не зависят от $x$, так и неравномерные оценки, которые стремятся к нулю при $|x| \rightarrow \infty$. (Различные равномерные и неравномерные оценки для разности функций распределения можно найти в [2].)

Мы получим верхние оценки величины $\left|p_{n}(x)-\varphi(x)\right|$, которые состоят из двух частей. Одна из них не зависит от $x$, но она убывает при росте $n$ быстрее, чем (неулучшаемые) равномерные оценки. Ее мы будем называть равномерной частью квазинеравномерной оценки. Вторая, которую мы будем называть неравномерной частью квазинеравномерной оценки, при росте $n$ убывает с той же скоростью, что (оптимальные

${ }^{*}$ Московский государственный университет им. М.В.Ломоносова, механикоматематический факультет, кафедра теории вероятностей, Ленинские горы, 119991 Москва, Россия; e-mail: v.senatov@yandex.ru

1) Работа выполнена при поддержке РФФИ (гранты № 08-01-00563, 09-01-1217 ОФИ-М). 
по $n$ ) равномерные и неравномерные оценки, но ее зависимость от $x$ в ряде случаев лучше, чем у (неулучшаемых по $x$ ) неравномерных оценок.

Мы будем предполагать, что для некоторого числа $\nu>0$

$$
\int_{-\infty}^{\infty}|f(t)|^{\nu} d t<\infty
$$

где $f$ - характеристическая функция распределения $P$. Выполнение этого условия гарантирует существование непрерывной и ограниченной плотности $p_{n}(x)$ при $n \geqslant \nu$, ее можно вычислять по формуле обращения

$$
p_{n}(x)=\frac{1}{2 \pi} \int_{-\infty}^{\infty} e^{-i t x} f^{n}\left(\frac{t}{\sqrt{n}}\right) d t, \quad-\infty<x<\infty .
$$

При выполнении условия (1) величина

$$
\alpha=\alpha(T)=\max \{|f(t)|: t \geqslant T\}
$$

строго меньше единицы для любого $T>0$.

Нам понадобится пара $(\mu, T)$, состоящая из функции $\mu(t), e^{-t^{2} / 2} \leqslant$ $\mu(t) \leqslant 1$, и числа $T>0$ такая, что

$$
|f(t)| \leqslant \mu(t) \text { для всех }|t| \leqslant T \text {. }
$$

С помощью пары $(\mu, T)$ введем числа

$$
B_{k, n-l}=\frac{1}{2 \pi} \int_{-T \sqrt{n}}^{T \sqrt{n}}|t|^{k} \mu^{n-l}\left(\frac{t}{\sqrt{n}}\right) d t,
$$

где $k>0, n \geqslant 2,0 \leqslant l \leqslant n$ (здесь, строго говоря, нужно использовать третий индекс, но мы этого делать не будем). О выборе пары $(\mu, T)$ подробно говорится в $[3$, гл. $4, \S 8]$. В случае $\beta_{3}<\infty$ в качестве пары $(\mu, T)$ можно взять $\mu(t)=e^{-t^{2} / 3}$ и $T=1 / \beta_{3}$. Для некоторых распределений $P$ (заведомо для любого распределения с конечным четвертым моментом) пару $(\mu, T)$ можно подобрать так, что $B_{k, n-l} \rightarrow \bar{\beta}_{k} / \sqrt{2 \pi}=B_{k}$ при $n \rightarrow \infty$ для любых фиксированных $k$ и $l$, где $\bar{\beta}_{k}$ - абсолютный момент порядка $k$ стандартного нормального закона.

Нам понадобятся многочлены Чебышёва-Эрмита

$$
H_{k}(x)=(-1)^{k} \frac{\varphi^{(k)}(x)}{\varphi(x)}, \quad-\infty<x<\infty, \quad k=0,1, \ldots,
$$

и функции

$$
\psi_{k}(x)=\max _{u \geqslant x}\left|H_{k}(u)\right| \varphi(u), \quad x>0 .
$$

Далее мы обозначаем $\alpha_{k}=\mathbf{E} X_{1}^{k}, k=0,1, \ldots$,

$$
\beta_{s}=\mathbf{E}\left|X_{1}\right|^{s}, \quad \beta_{s}^{+}=\int_{0}^{\infty} x^{s} P(d x), \quad s>0 .
$$


Оценки, предложенные ниже, не претендуют на роль окончательных результатов, но автор надеется, что они дадут некую почву для размышлений.

Теорема. Если четвертый момент распределения $P$ конечен, $\beta_{m^{\prime}+2}^{+}<\infty$ для некоторого $m^{\prime} \geqslant 2$ и выполнено условие (1), то при $n \geqslant \max (\nu, 2)$ для всех $x>0$

$$
\begin{aligned}
& \left|p_{n}(x)-\varphi(x)\right| \leqslant \frac{1}{2}\left(\frac{\beta_{3}+\bar{\beta}_{3}}{3 !}\right)^{2} \frac{B_{6, n-2}}{n}+\frac{\beta_{3}+\bar{\beta}_{3}}{12} \frac{\lambda^{6} B_{5}}{n^{3 / 2}} \\
& \quad+\frac{\left|\alpha_{3}\right|}{3 ! \sqrt{n}}\left|H_{3}(x)\right| \varphi(x)+\frac{\beta_{4}+3}{4 ! n} \psi_{4}(x / 2) \\
& \quad+\frac{2^{m^{\prime}+2} \beta_{m^{\prime}+2}^{+}}{\sqrt{2 \pi} n^{m^{\prime} / 2} x^{m^{\prime}+2}}+\frac{\beta_{m^{\prime}+2}^{+}+\bar{\beta}_{m^{\prime}+2} / 2}{n^{m^{\prime} / 2}} \sum_{k=0}^{3} \frac{\left|H_{k}(x)\right| \varphi(x)}{k !}\left(\frac{2}{x}\right)^{m^{\prime}+2-k} \\
& \quad+\frac{\sqrt{n}}{\pi} \alpha^{n-\nu} \int_{T}^{\infty}|f(t)|^{\nu} d t+\left(\frac{2 \sqrt{n}}{\pi T}+\frac{1}{\pi T \sqrt{n}}\right) e^{-T^{2} n / 2} \\
& \quad+\frac{\sqrt{n}}{\pi x} e^{-x^{2} n / 8} .
\end{aligned}
$$

При $n \geqslant \max (\nu, 3)$ справедливо также неравенство

$$
\begin{aligned}
\mid p_{n}(x) & -\varphi(x) \mid \leqslant \frac{1}{3 !}\left(\frac{\beta_{3}+\bar{\beta}_{3}}{3 !}\right)^{3} \frac{B_{9, n-3}}{n^{3 / 2}}+\frac{\beta_{3}+\bar{\beta}_{3}}{12} \frac{\lambda^{6} B_{5}}{n^{3 / 2}} \\
& +\frac{\left|\alpha_{3}\right|}{3 !} \frac{\beta_{4}+3}{4 !}\left(\frac{n}{n-2}\right)^{4} \frac{B_{7}}{n^{3 / 2}}+\frac{1}{2}\left(\frac{\beta_{4}+3}{4 !}\right)^{2}\left(\frac{n}{n-2}\right)^{9 / 2} \frac{B_{8}}{n^{2}} \\
& +\frac{\left|\alpha_{3}\right|}{3 ! \sqrt{n}}\left|H_{3}(x)\right| \varphi(x)+\frac{\beta_{4}+3}{4 ! n} \psi_{4}(x / 2) \\
& +\frac{1}{2}\left(\frac{n}{n-2}\right)^{7 / 2}\left(\frac{\alpha_{3}}{3 !}\right)^{2} \frac{1}{n}\left|H_{6}\left(\sqrt{\frac{n}{n-2}} x\right)\right| \varphi\left(\sqrt{\frac{n}{n-2}} x\right) \\
& +\frac{2^{m^{\prime}+2} \beta_{m^{\prime}+2}^{+}}{\sqrt{2 \pi} n^{m^{\prime} / 2} x^{m^{\prime}+2}}+\frac{\beta_{m^{\prime}+2}^{+}+\bar{\beta}_{m^{\prime}+2} / 2}{n^{m^{\prime} / 2}} \sum_{k=0}^{3} \frac{\left|H_{k}(x)\right| \varphi(x)}{k !}\left(\frac{2}{x}\right)^{m^{\prime}+2-k} \\
& +\frac{\sqrt{n}}{\pi} \alpha^{n-\nu} \int_{T}^{\infty}|f(t)|^{\nu} d t+\left(\frac{2 \sqrt{n}}{\pi T}+\frac{1}{\pi T \sqrt{n}}\right) e^{-T^{2} n / 2} \\
& +\frac{2 n^{3 / 2}}{\pi T} \frac{n}{n-2} e^{-T^{2}(n-2) / 2}+\frac{\sqrt{n}}{\pi x} e^{-x^{2} n / 8} \cdot
\end{aligned}
$$

Здесь $\lambda=\sqrt{n /(n-1)}$ и величина $T-$ та же, что в (2).

Отметим, что $H_{3}(x)=x^{3}-3 x$ и $\bar{\beta}_{3}=2 \sqrt{2 / \pi}$, число 3 в четвертом слагаемом правой части (3) совпадает с $\bar{\beta}_{4}$, величину $\bar{\beta}_{m^{\prime}+2}$ в шестом слагаемом правой части (3) можно заменить функцией, убывающей экспоненциально быстро при $x \sqrt{n} \rightarrow \infty$. Пятое слагаемое в правой части $(3)$ 
можно заменить величиной

$$
\frac{n}{\sqrt{2 \pi}}\left(1-F\left(\frac{x \sqrt{n}}{2}\right)\right)
$$

где $F$ - функция распределения меры $P$; если $F(u)=1$ для некоторого $u>0$, то это слагаемое равно нулю при $x \sqrt{n}>2 u$.

В теореме мы рассматриваем только положительные значения переменной $x$. Для того чтобы получить оценки при отрицательных $x$, достаточно перейти от случайных величин $X_{1}, X_{2}, \ldots$ к величинам $-X_{1},-X_{2}, \ldots$.

Формально квазинеравномерные оценки в ЦПТ можно получать из асимптотических разложений с явными оценками остаточных частей. Например, из теоремы 1, приведенной в [4], следует, что

$$
\left|p_{n}(x)-\varphi(x)\right| \leqslant \frac{\left|\alpha_{3}\right|}{3 ! \sqrt{n}}\left|H_{3}(x)\right| \varphi(x)+R .
$$

Явный вид оценки для $|R|$ указан в [4], одним из слагаемых этой оценки является величина $\left(\left(\beta_{4}+3\right) / 4 !\right)\left(B_{4, n-1} / n\right)$. Равномерные части квазинеравномерных оценок, получаемых таким путем, связаны с моментами максимальных порядков из тех, что используются в разложениях. В отличие от этого, в неравенстве (3), приведенном выше, равномерная часть квазинеравномерной оценки связана с абсолютным моментом третьего порядка, а момент четвертого порядка участвует в неравномерной части квазинеравномерной оценки.

Оценки нашей теоремы достаточно громоздки, но с помощью асимптотических разложений для плотностей $p_{n}$ нетрудно понять происхождение и тем самым отчасти оправдать появление некоторых слагаемых в этих оценках. Если у распределения $P$ существуют моменты достаточно высоких порядков и выполнено условие (1), то при больших $n$ для плотности $p_{n}$ справедливы асимптотические разложения, которые является суммами слагаемых, среди которых

$$
\begin{gathered}
\varphi(x), \quad \frac{\theta_{3}}{3 ! \sqrt{n}} H_{3}(x) \varphi(x), \quad \frac{\theta_{4}}{4 ! n} H_{4}(x) \varphi(x), \quad \frac{n-1}{2 n}\left(\frac{\theta_{3}}{3 !}\right)^{2} \frac{1}{n} H_{6}(x) \varphi(x), \\
\frac{\theta_{5}}{5 ! n^{3 / 2}} H_{5}(x) \varphi(x), \quad \frac{n-1}{n} \frac{\theta_{3}}{3 !} \frac{\theta_{4}}{4 !} \frac{1}{n^{3 / 2}} H_{7}(x) \varphi(x), \\
\frac{1}{3 !}\left(\frac{\theta_{3}}{3 !}\right)^{3} \frac{1}{n^{3 / 2}} H_{9}(x) \varphi(x), \quad \frac{1}{2}\left(\frac{\theta_{4}}{4 !}\right)^{2} \frac{1}{n^{2}} H_{8}(x) \varphi(x),
\end{gathered}
$$

где $\theta_{k}$ - моменты Чебышёва-Эрмита распределения $P$ (см. [3], [4]); отметим, что $\theta_{3}=\alpha_{3}$ и $\theta_{4}=\alpha_{4}-3$. Поэтому появление в правой части (3) третьего и четвертого слагаемых, а также появление в правой 
части (4) пятого, шестого и седьмого слагаемых совсем неудивительно. Величины $B_{k}$ являются оценками величин $\max _{-\infty<x<\infty}\left|H_{k}(x)\right| \varphi(x)$, поэтому столь же неудивительно появление первого слагаемого в правой части (3), а также первого, третьего и четвертого слагаемых в (4). Вторые слагаемые в $(3)$ и (4) дают оценки величины $\left|\alpha_{3}\right|\left|H_{5}(x)\right| \varphi(x) /\left(12 n^{3 / 2}\right)$ а число $\alpha_{3} / 12$ участвует в формировании величины $\theta_{5} / 5$ !, которая равна $\alpha_{5} / 5 !-\alpha_{3} / 12$.

Д о к а з а т е л с т в о т е о р е м ы. Очевидно, что при $n \geqslant \nu$

$$
\begin{aligned}
p_{n}(x)-\varphi(x) & =\frac{1}{2 \pi} \int_{-\infty}^{\infty} e^{-i t x}\left(f^{n}\left(\frac{t}{\sqrt{n}}\right)-g^{n}\left(\frac{t}{\sqrt{n}}\right)\right) d t \\
& =\frac{1}{2 \pi} \int_{-T \sqrt{n}}^{T \sqrt{n}} e^{-i t x}\left(f^{n}\left(\frac{t}{\sqrt{n}}\right)-g^{n}\left(\frac{t}{\sqrt{n}}\right)\right) d t+R,
\end{aligned}
$$

где $g(t)=e^{-t^{2} / 2}$, величина $T$ - та же, что в $(2)$, а

$$
\begin{aligned}
|R| & \leqslant \frac{1}{\pi} \int_{T \sqrt{n}}^{\infty}\left|f\left(\frac{t}{\sqrt{n}}\right)\right|^{n} d t+\frac{1}{\pi} \int_{T \sqrt{n}}^{\infty}\left|g\left(\frac{t}{\sqrt{n}}\right)\right|^{n} d t \\
& =\frac{\sqrt{n}}{\pi} \int_{T}^{\infty}|f(t)|^{n} d t+\frac{1}{\pi} \int_{T \sqrt{n}}^{\infty} e^{-t^{2} / 2} d t \\
& \leqslant \frac{\sqrt{n}}{\pi} \alpha^{n-\nu} \int_{T}^{\infty}|f(t)|^{\nu} d t+\frac{1}{\pi T \sqrt{n}} e^{-T^{2} n / 2} .
\end{aligned}
$$

Правая часть последнего неравенства при росте $n$ убывает экспоненциально быстро.

Дважды используя хорошо известное тождество

$$
a^{n}-b^{n}=\sum_{j=0}^{n-1} a^{n-j-1} b^{j}(a-b)
$$

справедливое для любого натурального $n$ и любых комплексных чисел $a$ и $b$, мы для $n \geqslant 2$ можем записать первое слагаемое в правой части (5) в виде

$$
\begin{gathered}
\sum_{j=0}^{n-1} \frac{1}{2 \pi} \int_{-T \sqrt{n}}^{T \sqrt{n}} e^{-i t x} f^{n-j-1}\left(\frac{t}{\sqrt{n}}\right) g^{j}\left(\frac{t}{\sqrt{n}}\right)\left(f\left(\frac{t}{\sqrt{n}}\right)-g\left(\frac{t}{\sqrt{n}}\right)\right) d t \\
=\sum_{j=0}^{n-2} \frac{1}{2 \pi} \int_{-T \sqrt{n}}^{T \sqrt{n}} e^{-i t x}\left(f^{n-j-1}\left(\frac{t}{\sqrt{n}}\right)-g^{n-j-1}\left(\frac{t}{\sqrt{n}}\right)\right) g^{j}\left(\frac{t}{\sqrt{n}}\right) \\
\times\left(f\left(\frac{t}{\sqrt{n}}\right)-g\left(\frac{t}{\sqrt{n}}\right)\right) d t \\
+\frac{n}{2 \pi} \int_{-T \sqrt{n}}^{T \sqrt{n}} e^{-i t x} g^{n-1}\left(\frac{t}{\sqrt{n}}\right)\left(f\left(\frac{t}{\sqrt{n}}\right)-g\left(\frac{t}{\sqrt{n}}\right)\right) d t
\end{gathered}
$$




$$
\begin{gathered}
=\sum_{j=0}^{n-2} \sum_{l=0}^{n-j-2} \frac{1}{2 \pi} \int_{-T \sqrt{n}}^{T \sqrt{n}} e^{-i t x} f^{n-j-l-2}\left(\frac{t}{\sqrt{n}}\right) g^{j+l}\left(\frac{t}{\sqrt{n}}\right) \\
\times\left(f\left(\frac{t}{\sqrt{n}}\right)-g\left(\frac{t}{\sqrt{n}}\right)\right)^{2} d t \\
+\frac{n}{2 \pi} \int_{-T \sqrt{n}}^{T \sqrt{n}} e^{-i t x} e^{-(n-1) t^{2} /(2 n)}\left(f\left(\frac{t}{\sqrt{n}}\right)-g\left(\frac{t}{\sqrt{n}}\right)\right) d t .
\end{gathered}
$$

Из формулы Тейлора с остаточным членом в интегральной форме следует, что при всех действительных $t$

$$
f\left(\frac{t}{\sqrt{n}}\right)=1-\frac{t^{2}}{2 n}+\gamma \frac{\beta_{3}}{3 !}\left(\frac{t}{\sqrt{n}}\right)^{3} .
$$

Здесь и далее мы используем одно и то же обозначение $\gamma$ для непрерывных комплекснозначных функций таких, что $|\gamma| \leqslant 1$. Аналогичное равенство, в котором $\beta_{3}$ заменяется на $\bar{\beta}_{3}$, справедливо и для $g(t / \sqrt{n})$. Поэтому при всех действительных $t$

$$
\left|f\left(\frac{t}{\sqrt{n}}\right)-g\left(\frac{t}{\sqrt{n}}\right)\right| \leqslant \frac{\beta_{3}+\bar{\beta}_{3}}{3 !}\left|\frac{t}{\sqrt{n}}\right|^{3}
$$

и мы видим, что абсолютная величина каждого слагаемого из двойной суммы в правой части (7) не превосходит

$$
\frac{1}{2 \pi} \int_{-T \sqrt{n}}^{T \sqrt{n}} \mu^{n-2}\left(\frac{t}{\sqrt{n}}\right)\left(\frac{\beta_{3}+\bar{\beta}_{3}}{3 !}\right)^{2}\left(\frac{t}{\sqrt{n}}\right)^{6} d t=\left(\frac{\beta_{3}+\bar{\beta}_{3}}{3 !}\right)^{2} \frac{B_{6, n-2}}{n^{3}}
$$

и абсолютная величина всей двойной суммы не превосходит

$$
\frac{n^{2}}{2}\left(\frac{\beta_{3}+\bar{\beta}_{3}}{3 !}\right)^{2} \frac{B_{6, n-2}}{n^{3}}
$$

Так как $\left|e^{t^{2} /(2 n)}-1\right| \leqslant t^{2}(2 n)^{-1} e^{t^{2} / 2 n}$ при всех действительных $t$, то последнее слагаемое в правой части (7) есть

$$
\begin{aligned}
& \frac{n}{2 \pi} \int_{-T \sqrt{n}}^{T \sqrt{n}} e^{-i t x} e^{t^{2} /(2 n)} e^{-t^{2} / 2}\left(f\left(\frac{t}{\sqrt{n}}\right)-g\left(\frac{t}{\sqrt{n}}\right)\right) d t \\
& \quad=\frac{n}{2 \pi} \int_{-\infty}^{\infty} e^{-i t x} e^{-t^{2} / 2}\left(f\left(\frac{t}{\sqrt{n}}\right)-g\left(\frac{t}{\sqrt{n}}\right)\right) d t+R_{1}+R_{2}
\end{aligned}
$$

где

$$
\left|R_{1}\right| \leqslant \frac{n}{2 \pi} \int_{-T \sqrt{n}}^{T \sqrt{n}} e^{-(n-1) t^{2} /(2 n)} \frac{t^{2}}{2 n} \frac{\beta_{3}+\bar{\beta}_{3}}{3 !}\left|\frac{t}{\sqrt{n}}\right|^{3} d t \leqslant \frac{\beta_{3}+\bar{\beta}_{3}}{12} \frac{\lambda^{6} B_{5}}{n^{3 / 2}}
$$

И

$$
\left|R_{2}\right| \leqslant \frac{n}{\pi} \int_{T \sqrt{n}}^{\infty} e^{-t^{2} / 2} \cdot 2 d t \leqslant \frac{2 \sqrt{n}}{\pi T} e^{-T^{2} n / 2}
$$


Из формулы обращения для преобразования Фурье следует, что $\frac{1}{2 \pi} \int_{-\infty}^{\infty} e^{-i t x} e^{-t^{2} / 2}\left(f\left(\frac{t}{\sqrt{n}}\right)-g\left(\frac{t}{\sqrt{n}}\right)\right) d t=\int_{-\infty}^{\infty} \varphi(x-y)\left(P_{1}-\Phi_{1}\right)(d y)$, где $P_{1}$ - распределение случайной величины $X_{1} / \sqrt{n}$, а $\Phi_{1}$ - нормальный закон с нулевым средним и дисперией $1 / n$.

Из сказанного следует, что

$$
p_{n}(x)-\varphi(x)=n \int_{-\infty}^{\infty} \varphi(x-y)\left(P_{1}-\Phi_{1}\right)(d y)+R,
$$

где

$$
\begin{aligned}
|R| \leqslant & \frac{1}{2}\left(\frac{\beta_{3}+\bar{\beta}_{3}}{3 !}\right)^{2} \frac{B_{6, n-2}}{n}+\frac{\beta_{3}+\bar{\beta}_{3}}{12} \frac{\lambda^{6} B_{5}}{n^{3 / 2}} \\
& +\frac{\sqrt{n}}{\pi} \alpha^{n-\nu} \int_{T}^{\infty}|f(t)|^{\eta} d t+\left(\frac{2 \sqrt{n}}{\pi T}+\frac{1}{\pi T \sqrt{n}}\right) e^{-T^{2} n / 2} .
\end{aligned}
$$

Правая часть последнего неравенства является равномерной частью квазинеравномерной оценки (3).

Рассмотрим первое слагаемое в правой части (8). Пусть $x>0$ и для некоторого целого числа $m \geqslant 2$ и числа $m^{\prime} \geqslant m$ величины

$$
\beta_{m+2}=\int_{-\infty}^{\infty}|u|^{m+2} P(d u) \quad \text { и } \quad \beta_{m^{\prime}+2}^{+}=\int_{0}^{\infty} u^{m^{\prime}+2} P(d u)
$$

конечны. По формуле Тейлора с остаточным членом в форме Лагранжа

$$
\begin{aligned}
\varphi(x-y) & =\sum_{k=0}^{m+1} \frac{\varphi^{(k)}(x)}{k !}(-y)^{k}+\frac{\varphi^{(m+2)}(x-\theta y)}{(m+2) !}(-y)^{m+2} \\
& =\sum_{k=0}^{m+1} \frac{H_{k}(x) \varphi(x)}{k !} y^{k}+\frac{H_{m+2}(x-\theta y) \varphi(x-\theta y)}{(m+2) !} y^{m+2},
\end{aligned}
$$

где $0 \leqslant \theta \leqslant 1$. Поэтому

$$
\begin{aligned}
n & \int_{-\infty}^{x / 2} \varphi(x-y)\left(P_{1}-\Phi_{1}\right)(d y) \\
= & n \sum_{k=0}^{m+1} \frac{H_{k}(x) \varphi(x)}{k !} \int_{-\infty}^{x / 2} y^{k}\left(P_{1}-\Phi_{1}\right)(d y) \\
& +n \int_{-\infty}^{x / 2} \frac{H_{m+2}(x-\theta y) \varphi(x-\theta y)}{(m+2) !} y^{m+2}\left(P_{1}-\Phi_{1}\right)(d y) \\
= & n \sum_{k=3}^{m+1} \frac{H_{k}(x) \varphi(x)}{k !} \frac{\Delta_{k}}{n^{k / 2}} \\
& -\left\{n \sum_{k=0}^{m+1} \frac{H_{k}(x) \varphi(x)}{k !} \int_{x / 2}^{\infty} y^{k}\left(P_{1}-\Phi_{1}\right)(d y)\right\}+R,
\end{aligned}
$$


где $\Delta_{k}$ - разность моментов порядка $k$ распределений $P$ и $\Phi$ (отсутствие в первой сумме правой части последнего равенства слагаемых, соответствующих $k=0,1$ и 2 , связано с тем, что $\int_{-\infty}^{\infty} P(d y)=\int_{-\infty}^{\infty} \Phi(d y)$ и первые два момента распределений $P$ и $\Phi$ совпадают), а

$$
|R| \leqslant n \frac{\psi_{m+2}(x / 2)}{(m+2) !} \int_{-\infty}^{x / 2}|y|^{m+2}\left(P_{1}+\Phi_{1}\right)(d y) \leqslant \frac{\beta_{m+2}+\bar{\beta}_{m+2}}{(m+2) ! n^{m / 2}} \psi_{m+2}\left(\frac{x}{2}\right) .
$$

Модуль выражения в фигурных скобках в (9) можно оценить величиной

$$
\begin{gathered}
n \sum_{k=0}^{m+1} \frac{\left|H_{k}(x)\right| \varphi(x)}{k !}\left(\frac{2}{x}\right)^{m^{\prime}+2-k} \int_{x / 2}^{\infty} y^{m^{\prime}+2}\left(P_{1}+\Phi_{1}\right)(d y) \\
\leqslant \frac{\beta_{m^{\prime}+2}^{+}+\bar{\beta}_{m^{\prime}+2} / 2}{n^{m^{\prime} / 2}} \sum_{k=0}^{m+1} \frac{\left|H_{k}(x)\right| \varphi(x)}{k !}\left(\frac{2}{x}\right)^{m^{\prime}+2-k} .
\end{gathered}
$$

Нам осталось рассмотреть величину

$$
n \int_{x / 2}^{\infty} \varphi(x-y)\left(P_{1}-\Phi_{1}\right)(d y)
$$

Ее модуль не превосходит

$$
\begin{aligned}
\frac{n}{\sqrt{2 \pi}} \int_{x \sqrt{n} / 2}^{\infty}(P+\Phi)(d y) & \leqslant \frac{n}{\sqrt{2 \pi}}\left(\frac{2}{x \sqrt{n}}\right)^{m^{\prime}+2} \beta_{m^{\prime}+2}^{+}+\frac{n}{\sqrt{2 \pi}} \int_{x \sqrt{n} / 2}^{\infty} \varphi(y) d y \\
& \leqslant \frac{2^{m^{\prime}+2} \beta_{m^{\prime}+2}^{+}}{\sqrt{2 \pi} n^{m^{\prime} / 2} x^{m^{\prime}+2}}+\frac{\sqrt{n}}{\pi x} e^{-x^{2} n / 8} .
\end{aligned}
$$

Из сказанного следует, что первое слагаемое в правой части (8) при $m=2$ (т.е. при конечности момента $\beta_{4}$ ) и при $m^{\prime} \geqslant 2$ есть

$$
\frac{\alpha_{3}}{3 ! \sqrt{n}} H_{3}(x) \varphi(x)+R
$$

где

$$
\begin{aligned}
|R| \leqslant & \frac{\beta_{4}+3}{4 ! n} \psi_{4}\left(\frac{x}{2}\right)+\frac{\beta_{m^{\prime}+2}^{+}+\bar{\beta}_{m^{\prime}+2} / 2}{n^{m^{\prime} / 2}} \sum_{k=0}^{3} \frac{\left|H_{k}(x)\right| \varphi(x)}{k !}\left(\frac{2}{x}\right)^{m^{\prime}+2-k} \\
& +\frac{2^{m^{\prime}+2} \beta_{m^{\prime}+2}^{+}}{\sqrt{2 \pi} n^{m^{\prime} / 2} x^{m^{\prime}+2}}+\frac{\sqrt{n}}{\pi x} e^{-x^{2} n / 8}
\end{aligned}
$$

Собирая вместе полученные оценки, мы убеждаемся в справедливости неравенства (3).

Для того чтобы доказать неравенство (4), перепишем двойную сумму из (7) в виде

$$
\sum_{j=0}^{n-2} \frac{j+1}{2 \pi} \int_{-T \sqrt{n}}^{T \sqrt{n}} e^{-i t x} f^{n-j-2}\left(\frac{t}{\sqrt{n}}\right) g^{j}\left(\frac{t}{\sqrt{n}}\right)\left(f\left(\frac{t}{\sqrt{n}}-g\left(\frac{t}{\sqrt{n}}\right)\right)^{2} d t .\right.
$$


Применяя еще раз тождество (6) (напомним, что $n \geqslant 3$ ), мы видим, что эта сумма равна

$$
\begin{gathered}
\sum_{j=0}^{n-3} \frac{j+1}{2 \pi} \int_{-T \sqrt{n}}^{T \sqrt{n}} e^{-i t x}\left(f^{n-j-2}\left(\frac{t}{\sqrt{n}}\right)-g^{n-j-2}\left(\frac{t}{\sqrt{n}}\right)\right) g^{j}\left(\frac{t}{\sqrt{n}}\right) \\
\times\left(f\left(\frac{t}{\sqrt{n}}\right)-g\left(\frac{t}{\sqrt{n}}\right)\right)^{2} d t \\
\quad+\sum_{j=0}^{n-2} \frac{j+1}{2 \pi} \int_{-T \sqrt{n}}^{T \sqrt{n}} e^{-i t x} g^{n-2}\left(\frac{t}{\sqrt{n}}\right)\left(f\left(\frac{t}{\sqrt{n}}\right)-g\left(\frac{t}{\sqrt{n}}\right)\right)^{2} d t \\
=\sum_{j=0}^{n-3} \frac{j+1}{2 \pi} \sum_{l=0}^{n-j-3} \int_{-T \sqrt{n}}^{T \sqrt{n}} e^{-i t x} f^{n-j-l-3}\left(\frac{t}{\sqrt{n}}\right) g^{j+l}\left(\frac{t}{\sqrt{n}}\right) \\
\quad \times\left(f\left(\frac{t}{\sqrt{n}}\right)-g\left(\frac{t}{\sqrt{n}}\right)\right)^{3} d t \\
+C_{n}^{2} \frac{1}{2 \pi} \int_{-T \sqrt{n}}^{T \sqrt{n}} e^{-i t x} g^{n-2}\left(\frac{t}{\sqrt{n}}\right)\left(f\left(\frac{t}{\sqrt{n}}\right)-g\left(\frac{t}{\sqrt{n}}\right)\right)^{2} d t .
\end{gathered}
$$

Используя полученную ранее оценку для $|f(t / \sqrt{n})-g(t / \sqrt{n})|$, мы видим, что абсолютная величина правой части этого равенства, за исключением последнего слагаемого, не превосходит

$$
C_{n}^{3}\left(\frac{\beta_{3}+\bar{\beta}_{3}}{3 !}\right)^{3} \frac{B_{9, n-3}}{n^{9 / 2}} .
$$

Последнее же слагаемое в правой части этого равенства есть

$$
C_{n}^{2} \frac{1}{2 \pi} \int_{-\infty}^{\infty} e^{-i t x} g^{n-2}\left(\frac{t}{\sqrt{n}}\right)\left(f\left(\frac{t}{\sqrt{n}}\right)-g\left(\frac{t}{\sqrt{n}}\right)\right)^{2} d t+R
$$

где

$$
|R| \leqslant C_{n}^{2} \frac{1}{\pi} \int_{T \sqrt{n}}^{\infty} e^{-(n-2) t^{2} /(2 n)} \cdot 4 d t \leqslant \frac{2 n^{3 / 2}}{\pi T} \frac{n}{n-2} e^{-(n-2) T^{2} / 2} .
$$

Рассмотрим первое слагаемое в (11). Так как

$$
f\left(\frac{t}{\sqrt{n}}\right)-g\left(\frac{t}{\sqrt{n}}\right)=\frac{\alpha_{3}}{3 !}\left(\frac{i t}{\sqrt{n}}\right)^{3}+\gamma \frac{\beta_{4}+3}{4 !}\left(\frac{t}{\sqrt{n}}\right)^{4}
$$

то оно равно

$$
C_{n}^{2} \frac{1}{2 \pi} \int_{-\infty}^{\infty} e^{-i t x} e^{-(n-2) t^{2} /(2 n)}\left(\frac{\alpha_{3}}{3 !}\right)^{2}\left(\frac{i t}{\sqrt{n}}\right)^{6} d t+R,
$$


где

$$
\begin{aligned}
|R| & \leqslant C_{n}^{2} \frac{1}{2 \pi} \int_{-\infty}^{\infty} e^{-(n-2) t^{2} /(2 n)}\left(2 \frac{\left|\alpha_{3}\right|}{3 !} \frac{\beta_{4}+3}{4 !}\left|\frac{t}{\sqrt{n}}\right|^{7}+\left(\frac{\beta_{4}+3}{4 !}\right)^{2}\left(\frac{t}{\sqrt{n}}\right)^{8}\right) d t \\
& \leqslant \frac{\left|\alpha_{3}\right|}{3 !} \frac{\beta_{4}+3}{4 !}\left(\frac{n}{n-2}\right)^{4} \frac{B_{7}}{n^{3 / 2}}+\frac{1}{2}\left(\frac{\beta_{4}+3}{4 !}\right)^{2}\left(\frac{n}{n-2}\right)^{9 / 2} \frac{B_{8}}{n^{2}}
\end{aligned}
$$

Для любого целого неотрицательного $k$ справедлива формула обращения

$$
\frac{1}{2 \pi} \int_{-\infty}^{\infty} e^{-i t x} e^{-t^{2} / 2}(i t)^{k} d t=H_{k}(x) \varphi(x)
$$

поэтому первое слагаемое в (12) равно

$$
\frac{n-1}{2 n}\left(\frac{n}{n-2}\right)^{7 / 2}\left(\frac{\alpha_{3}}{3 !}\right)^{2} \frac{1}{n} H_{6}\left(\sqrt{\frac{n}{n-2}} x\right) \varphi\left(\sqrt{\frac{n}{n-2}} x\right) .
$$

Теперь, собирая вместе полученные оценки, нетрудно убедиться в справедливости неравенства (4).

Для первого слагаемого в (11) можно получить и другую оценку. Она существенно точнее той, что только что доказана (во всяком случае, при больших $n$ ), однако имеет значительно более громоздкий вид. Поэтому мы дадим лишь набросок ее доказательства и для простоты (чтобы не появлялись многочисленные множители, связанные со степенями числа $n /(n-2))$ оценим величину

$$
\frac{1}{2 \pi} \int_{-\infty}^{\infty} e^{-i t x} e^{-t^{2} / 2}\left(f\left(\frac{t}{\sqrt{n}}\right)-g\left(\frac{t}{\sqrt{n}}\right)\right)^{2} d t
$$

которая равна

$$
\int_{-\infty}^{\infty} \int_{-\infty}^{\infty} \varphi(x-y-z)\left(P_{1}-\Phi_{1}\right)(d y)\left(P_{1}-\Phi_{1}\right)(d z) .
$$

Введем множества $A_{1}=\{y<x / 3, z<x / 3\}, A_{2}=\{-\infty<y<$ $\infty, z \geqslant x / 3\}, A_{3}=\{y \geqslant x / 3,-\infty<z<\infty\}, A_{4}=\{y>x / 3, z>x / 3\}$. Очевидно, что для индикаторов этих множеств справедливо тождество

$$
\chi_{A_{1}}(y, z)+\chi_{A_{2}}(y, z)+\chi_{A_{3}}(y, z)-\chi_{A_{4}}(y, z) \equiv 1, \quad-\infty<y, z<\infty,
$$

поэтому нам достаточно оценить абсолютные величины каждого из интегралов

$$
\iint_{A_{k}} \varphi(x-y-z)\left(P_{1}-\Phi_{1}\right)(d y)\left(P_{1}-\Phi_{1}\right)(d z), \quad k=1,2,3,4 .
$$

Рассмотрим интеграл по множеству $A_{1}$, который равен

$$
\int_{-\infty}^{x / 3} U(z)\left(P_{1}-\Phi_{1}\right)(d z)
$$


где $U(z)=\int_{-\infty}^{x / 3} \varphi(x-y-z)\left(P_{1}-\Phi_{1}\right)(d y)$. Очевидно, что

$$
\begin{aligned}
& \int_{-\infty}^{x / 3} U(z)\left(P_{1}-\Phi_{1}\right)(d z) \\
& =\int_{-\infty}^{x / 3}\left(U(0)+U^{\prime}(0) z+\frac{1}{2} U^{\prime \prime}(0) z^{2}+\frac{1}{3 !} U^{\prime \prime \prime}(0) z^{3}\right)\left(P_{1}-\Phi_{1}\right)(d z) \\
& \quad+\frac{1}{4 !} \int_{-\infty}^{x / 3} U^{(4)}\left(\theta_{2} z\right) z^{4}\left(P_{1}-\Phi_{1}\right)(d z),
\end{aligned}
$$

где $0 \leqslant \theta_{2} \leqslant 1$. Последнее слагаемое равно

$$
\begin{aligned}
\frac{1}{4 !} \int_{-\infty}^{x / 3}\left\{\int_{-\infty}^{x / 3} \varphi^{(4)}\left(x-y-\theta_{2} z\right)\left(P_{1}-\Phi_{1}\right)(d y)\right\} z^{4}\left(P_{1}-\Phi_{1}\right)(d z) \\
=\frac{1}{4 !} \int_{-\infty}^{x / 3}\left\{\int_{-\infty}^{x / 3}\left(V(0)+V^{\prime}(0) y+\frac{1}{2} V^{\prime \prime}(0) y^{2}+\frac{1}{6} V^{\prime \prime \prime}(0) y^{3}\right)\right. \\
\left.\times\left(P_{1}-\Phi_{1}\right)(d y)\right\} z^{4}\left(P_{1}-\Phi_{1}\right)(d z) \\
+\left(\frac{1}{4 !}\right)^{2} \int_{-\infty}^{x / 3}\left\{\int_{-\infty}^{x / 3} V^{(4)}\left(\theta_{1} y\right) y^{4}\left(P_{1}-\Phi_{1}\right)(d y)\right\} z^{4}\left(P_{1}-\Phi_{1}\right)(d z),
\end{aligned}
$$

где $V(y)=\varphi^{(4)}\left(x-y-\theta_{2} z\right)$ и $0 \leqslant \theta_{1} \leqslant 1$. Абсолютная величина последнего интеграла не превосходит

$$
\begin{aligned}
& \int_{-\infty}^{x / 3}\left\{\int_{-\infty}^{x / 3}\left|\varphi^{(8)}\left(x-\theta_{1} y-\theta_{2} z\right)\right| y^{4}\left(P_{1}+\Phi_{1}\right)(d y)\right\} z^{4}\left(P_{1}+\Phi_{1}\right)(d z) \\
& \quad \leqslant \frac{\left(\beta_{4}+3\right)^{2}}{n^{4}} \psi_{8}(x / 3) .
\end{aligned}
$$

Очевидно, что

$$
\begin{aligned}
& \int_{-\infty}^{x / 3} V^{\prime \prime \prime}(0)\left\{\int_{-\infty}^{x / 3} y^{3}\left(P_{1}-\Phi_{1}\right)(d y)\right\} z^{4}\left(P_{1}-\Phi_{1}\right)(d z) \\
& =\int_{-\infty}^{x / 3} H_{7}\left(x-\theta_{2} z\right) \varphi\left(x-\theta_{2} z\right)\left\{\int_{-\infty}^{\infty}\left(P_{1}-\Phi_{1}\right)(d y)\right\} z^{4}\left(P_{1}-\Phi_{1}\right)(d z) \\
& \quad-\int_{-\infty}^{x / 3} H_{7}\left(x-\theta_{2} z\right) \varphi\left(x-\theta_{2} z\right)\left\{\int_{x / 3}^{\infty} y^{3}\left(P_{1}-\Phi_{1}\right)(d y)\right\} z^{4}\left(P_{1}-\Phi_{1}\right)(d z) .
\end{aligned}
$$

Абсолютная величина правой части последнего равенства не превосходит

$$
\begin{aligned}
& \frac{\left|\alpha_{3}\right|}{n^{3 / 2}} \psi_{7}\left(\frac{2 x}{3}\right) \int_{-\infty}^{x / 3} z^{4}\left(P_{1}+\Phi_{1}\right)(d z) \\
& \quad+\psi_{7}\left(\frac{2 x}{3}\right) \frac{\beta_{m^{\prime}+2}^{+}+\bar{\beta}_{m^{\prime}+2} / 2}{n^{\left(m^{\prime}+2\right) / 2}}\left(\frac{3}{x}\right)^{m^{\prime}-1} \int_{-\infty}^{x / 3} z^{4}\left(P_{1}+\Phi_{1}\right)(d z) \\
& \leqslant \frac{\left|\alpha_{3}\right|}{n^{3 / 2}} \frac{\beta_{4}+3}{n^{2}} \psi_{7}\left(\frac{2 x}{3}\right)+\frac{\beta_{4}+3}{n^{2}} \frac{\beta_{m^{\prime}+2}^{+}+\bar{\beta}_{m^{\prime}+2} / 2}{n^{\left(m^{\prime}+2\right) / 2}}\left(\frac{3}{x}\right)^{m^{\prime}-1} \psi_{7}\left(\frac{2 x}{3}\right) .
\end{aligned}
$$


Очевидно, что

$$
\begin{aligned}
& \int_{-\infty}^{x / 3} V^{\prime \prime}(0)\left\{\int_{-\infty}^{x / 3} y^{2}\left(P_{1}-\Phi_{1}\right)(d y)\right\} z^{4}\left(P_{1}-\Phi_{1}\right)(d z) \\
& =-\int_{-\infty}^{x / 3} H_{6}\left(x-\theta_{2} z\right) \varphi\left(x-\theta_{2} z\right)\left\{\int_{x / 3}^{\infty} y^{2}\left(P_{1}-\Phi_{1}\right)(d y)\right\} z^{4}\left(P_{1}-\Phi_{1}\right)(d z) .
\end{aligned}
$$

Абсолютная величина правой части этого равенства не превосходит

$$
\frac{\beta_{4}+3}{n^{2}} \frac{\beta_{m^{\prime}+2}^{+}+\bar{\beta}_{m^{\prime}+2} / 2}{n^{\left(m^{\prime}+2\right) / 2}}\left(\frac{3}{x}\right)^{m^{\prime}} \psi_{6}\left(\frac{2 x}{3}\right) \text {. }
$$

Интегралы

$$
\int_{-\infty}^{x / 3} V^{\prime}(0)\left\{\int_{-\infty}^{x / 3} y\left(P_{1}-\Phi_{1}\right)(d y)\right\} z^{4}\left(P_{1}-\Phi_{1}\right)(d z)
$$

и

$$
\int_{-\infty}^{x / 3} V(0)\left\{\int_{-\infty}^{x / 3}\left(P_{1}-\Phi_{1}\right)(d y)\right\} z^{4}\left(P_{1}-\Phi_{1}\right)(d z)
$$

рассматриваются аналогично.

Займемся теперь интегралами

$$
\int_{-\infty}^{x / 3} U^{(k)}(0) z^{k}\left(P_{1}-\Phi_{1}\right)(d z), \quad k=0,1,2,3
$$

Их абсолютные величины не превосходят произведений величин $\left|U^{(k)}(0)\right|$ и $\left|\int_{-\infty}^{x / 3} z^{k}\left(P_{1}-\Phi_{1}\right)(d z)\right|$.

Ясно, что

$$
\left|\int_{-\infty}^{x / 3} z^{k}\left(P_{1}-\Phi_{1}\right)(d z)\right| \leqslant\left|\int_{-\infty}^{\infty} z^{k}\left(P_{1}-\Phi_{1}\right)(d z)\right|+\int_{x / 3}^{\infty} z^{k}\left(P_{1}+\Phi_{1}\right)(d z) .
$$

Первое слагаемое в правой части этого неравенства есть $\left|\alpha_{3}\right| / n^{3 / 2}$ для $k=3$ и нуль для $k=0,1,2$, а второе слагаемое не превосходит

$$
\frac{\beta_{m^{\prime}+2}^{+}+\bar{\beta}_{m^{\prime}+2} / 2}{n^{\left(m^{\prime}+2\right) / 2}}\left(\frac{3}{x}\right)^{m^{\prime}+2-k}
$$

для $k=0,1,2,3$.

Вновь воспользовавшись формулой Тейлора с остаточным членом в форме Лагранжа, мы видим, что

$$
\begin{aligned}
\left|U^{(k)}(0)\right|= & \left|\int_{-\infty}^{x / 3} H_{k}(x-y) \varphi(x-y)\left(P_{1}-\Phi_{1}\right)(d y)\right| \\
\leqslant & \sum_{l=0}^{3} \frac{1}{l !}\left|H_{k+l}(x)\right| \varphi(x)\left|\int_{-\infty}^{x / 3} y^{l}\left(P_{1}-\Phi_{1}\right)(d y)\right| \\
& +\frac{1}{4 !}\left|\int_{-\infty}^{x / 3} H_{k+4}(x-\theta y) \varphi(x-\theta y) y^{4}\left(P_{1}-\Phi_{1}\right)(d y)\right| .
\end{aligned}
$$


Оценка суммы по $l=0,1,2,3$ не вызывает трудностей. Последнее слагаемое в правой части последнего неравенства не превосходит

$$
\frac{1}{4 !} \frac{\beta_{4}+3}{n^{2}} \psi_{k+4}\left(\frac{2 x}{3}\right) \text {. }
$$

Теперь нетрудно выписать оценку для абсолютной величины интеграла по множеству $A_{1}$ (ничего, кроме терпения, при этом не понадобится), но мы этого делать не будем.

Рассмотрим интеграл

$$
\begin{aligned}
& \iint_{A_{2}} \varphi(x-y-z)\left(P_{1}-\Phi_{1}\right)(d y)\left(P_{1}-\Phi_{1}\right)(d z) \\
& =\int_{x / 3}^{\infty}\left\{\int_{-\infty}^{\infty} \varphi(x-y-z)\left(P_{1}-\Phi_{1}\right)(d y)\right\}\left(P_{1}-\Phi_{1}\right)(d z) .
\end{aligned}
$$

Функция $\varphi(x-y-z)$ переменной $y$ является бесконечно дифференцируемой и абсолютная величина ее третьей производной не превосходит $\max _{-\infty<x<\infty}\left|H_{3}(x)\right| \varphi(x)=c_{3} / \sqrt{2 \pi}$, где $c_{3}=1,27 \ldots$ (указанный максимум достигается в точках $\pm(3-\sqrt{6}))$. Поэтому

$$
\left|\int_{-\infty}^{\infty} \varphi(x-y-z)\left(P_{1}-\Phi_{1}\right)(d y)\right| \leqslant \frac{c_{3}}{\sqrt{2 \pi}} \zeta_{3}\left(P_{1}, \Phi_{1}\right) \leqslant \frac{c_{3}}{\sqrt{2 \pi}} \frac{\beta_{3}+\bar{\beta}_{3}}{3 ! n^{3 / 2}}
$$

(идеальные метрики $\zeta_{s}, s>0$, введены и исследованы В. М. Золотаревым, см., например [1], [3]). Отсюда следует, что

$$
\begin{aligned}
& \left|\iint_{A_{2}} \varphi(x-y-z)\left(P_{1}-\Phi_{1}\right)(d y)\left(P_{1}-\Phi_{1}\right)(d z)\right| \\
& \quad \leqslant \frac{c_{3}}{\sqrt{2 \pi}} \frac{\beta_{3}+\bar{\beta}_{3}}{3 ! n^{3 / 2}}\left(\frac{\beta_{m^{\prime}+2}^{+}}{n^{\left(m^{\prime}+2\right) / 2}}+\frac{1}{\sqrt{2 \pi}} \frac{3}{x \sqrt{n}} e^{-x^{2} n / 8}\right) .
\end{aligned}
$$

Эта же оценка справедлива и для интеграла по множеству $A_{3}$.

Очевидно, что

$$
\begin{aligned}
& \left|\iint_{A_{4}} \varphi(x-y-z)\left(P_{1}-\Phi_{1}\right)(d y)\left(P_{1}-\Phi_{1}\right)(d z)\right| \\
& \quad \leqslant \frac{1}{\sqrt{2 \pi}}\left(\int_{x / 3}^{\infty}\left(P_{1}+\Phi_{1}\right)(d y)\right)^{2} \\
& \quad \leqslant \frac{1}{\sqrt{2 \pi}}\left(\frac{\beta_{m^{\prime}+2}^{+}}{n^{\left(m^{\prime}+2\right) / 2}}\left(\frac{3}{x}\right)^{m^{\prime}+2}+\frac{3}{x \sqrt{2 \pi n}} e^{-x^{2} n / 18}\right)^{2} .
\end{aligned}
$$

Теперь нетрудно выписать новую (неравномерную) оценку для первого слагаемого в (11), но мы этого делать не будем. 
Отметим, что при доказательстве теоремы мы получили асимптотическое разложение для плотности

$$
p_{n}(x)=\varphi(x)+\frac{\alpha_{3}}{3 ! \sqrt{n}} H_{3}(x) \varphi(x)+R
$$

и для величины $R=O(1 / n), n \rightarrow \infty$, из неравенства (4) легко получается квазинеравномерная оценка для этого разложения. Эта оценка отличается от правой части (4) лишь тем, что в ней нет слагаемого $\left(\left|\alpha_{3}\right| /(3 ! \sqrt{n})\right)\left|H_{3}(x)\right| \varphi(x)$.

\section{СПИСОК ЛИТЕРАТУРЫ}

1. Золотарев В. М. Современная теория суммирования независимых случайных величин. М.: Наука, 1986, 416 с.

2. Петров В. В. Суммы независимых случайных величин. М.: Наука, 1972, 414 с.

3. Сенатов B. В. Центральная предельная теорема: точность аппроксимации и асимптотические разложения. М.: Книжный дом «Либроком», 2009, 352 с.

4. Сенатов В.В. Об асимптотических разложениях в центральной предельной теореме с явными оценками остаточных членов. - Теория вероятн. и ее примен., 2006, т. 51, в. 4 , с. $810-816$.

Поступила в редакцию 27.VII.2008 